Research Article

\title{
Quantification of Dynamic Properties of Pile Using Ensemble Empirical Mode Decomposition
}

\author{
Feng Xiao $\mathbb{D}^{1},{ }^{1}$ Gang S. Chen $\mathbb{D}^{2},{ }^{2}$ Wael Zatar, ${ }^{2}$ and J. Leroy Hulsey ${ }^{3}$ \\ ${ }^{1}$ Department of Civil Engineering, Nanjing University of Science and Technology, Nanjing, China \\ ${ }^{2}$ College of Information Technology and Engineering, Marshall University, Huntington, WV, USA \\ ${ }^{3}$ Department of Civil and Environmental Engineering, University of Alaska Fairbanks, Fairbanks, AK, USA
}

Correspondence should be addressed to Gang S. Chen; chenga@marshall.edu

Received 28 August 2017; Revised 31 December 2017; Accepted 18 January 2018; Published 11 March 2018

Academic Editor: Claudio Tamagnini

Copyright (C) 2018 Feng Xiao et al. This is an open access article distributed under the Creative Commons Attribution License, which permits unrestricted use, distribution, and reproduction in any medium, provided the original work is properly cited.

This paper investigated dynamical interactions between pile and frozen ground by using the ensemble empirical mode decomposition (EEMD) method. Unlike the conventional empirical mode decomposition (EMD) method, EEMD is found to be able to separate the mode patterns of pile response signals of different scales without causing mode mixing. The identified dynamic properties using the EEMD method are more accurate than those obtained from conventional methods. EEMD-based results can be used to reliably and accurately characterize pile-frozen soil interactions and help designing infrastructure foundations under permafrost condition.

\section{Introduction}

To quantify dynamical properties of structures, timefrequency analysis can be used to effectively reveal the constituent frequency components of nonstationary signals and their time variation features, as well as transient events such as impulses. To date, various time-frequency analysis methods have been proposed $[1,2]$. However, the inherent drawbacks of these time-frequency analysis methods limit their effectiveness in analyzing complex vibration signals. For instance, linear transforms such as the short time Fourier transform (STFT) and wavelet transform are subject to the Heisenberg uncertainty principle, that is, the best time localization and highest frequency resolution cannot be achieved simultaneously. One of them can only be enhanced at the expense of the other, and hence, the time-frequency resolutions of linear transforms are limited. In addition, the basis in either the Fourier or wavelet transform is fixed. Therefore, the conventional methods lack adaptability in simultaneously matching the complicated components inherent in complex vibration signals, such as the fundamental frequency and their harmonics, combined harmonics, chaos, system-induced impulses, and other transient vibration. As a typical representative of bilinear time-frequency representations,
Wigner-Ville distribution has the best time-frequency resolution, but it has the inevitable cross-term interferences for multiple component signals. Such cross-term interferences complicate the interpretation of signal features in the timefrequency domain and make it unsuitable to analyzing the complex vibration signals. Various modified bilinear timefrequency distributions including Cohen and affine class distributions may suppress the negative effect of cross-terms but will compromise time-frequency resolution and autoterm integrity. Spectrogram better preserves energy distribution (comparing to wavelet transform), but it has issues with time/frequency resolution and gives rather rough estimates. Wavelet transform and scalograms provide good results but add artifacts on frequencies close to zero or close to the edges. Also, the conventional methods distort energy distribution with high-frequency components having less power comparing to lower ones.

The well-known Hilbert-Huang transform has fine timefrequency resolution and is free of cross-term interferences, but it essentially relies on the empirical mode decomposition (EMD) using spline interpolation [2]. It is susceptible to singularities in signals, and may produce pseudointrinsic mode functions (IMF), thus masking or interfering the timefrequency structure of true signal components. Oscillations 
of different amplitudes are found in a mode, or similar oscillations are encountered in different modes. To avoid this problem, $\mathrm{Wu}$ and Huang proposed ensemble EMD (EEMD) [3], a method based on the EMD algorithm. The proposed method follows a study of the statistical characteristics of white noise, involves a noise-assisted analysis, and adds white noise of a uniform frequency distribution into EMD to avoid mode mixing.

In the Northern area, there are some bridges damaged directly due to the occurrence of frozen ground. Recently, some research has identified that the effects of frozen ground on seismic response of bridge foundations are significant, which has validated the requirements for design guidelines to include the consideration of the frozen ground effects [4-7]. Seasonally frozen ground across the Northern area is significantly stiffer than unfrozen ground. For bridges supported on deep foundations, the overall bridge stiffness increases measurably in winter season [7]. As bridge stiffness increases, seismic demand increases. To address significant changes in bridge pier boundary conditions, additional knowledge of the subsurface is needed. Extra information is needed to address a bridge's ductile performance in bridge design for earthquake prevention event. The study of the effects of frozen ground on foundation has been required for bridge designers. Several studies have been performed to evaluate the soil-foundation-structure interaction $[4,5,8,9]$. There always has been a need for engineers to reliably and accurately quantify dynamical interactions between foundation and frozen ground by using the latest method.

As a relatively new technique, EEMD has been successfully applied in some engineering problems. For example, varied fault patterns in machine bearings have been identified using EEMD, whereas these fault frequencies could not be identified using fast Fourier transform (FFT) [10]. In [11], EEMD is applied to extract specific components from a data set of passenger flows of a station in the metro system and illustrates the time variants of short-term passenger flow for these data sets. The EEMD method is used to analyze carbon price pattern and extract carbon price signals from a multiscale viewpoint [12]. Also, EEMD is used for trend prediction of market indexes [13].

In this study, the pile dynamic behavior in frozen ground condition has been determined by using the EEMD method. A test pile was constructed and tested for free vibration in winter conditions in Alaska to assess the effects of frozen soils on the performance of single piles. To accurately quantify the dynamic properties of pile on frozen soil, we use EEMD to decompose the transient response of pile into serial components and then use these components to identify the system dynamic properties including natural frequencies and mode damping. The study provided a basis for accurately and reliably analyzing the frozen ground effects on a pile structure and for designing bridge foundations.

\section{Test Setup and Measurements}

The test site is at mile 0.9 Farmers Loop Road at Fairbanks in Alaska. Some mechanical properties of soil at the test site

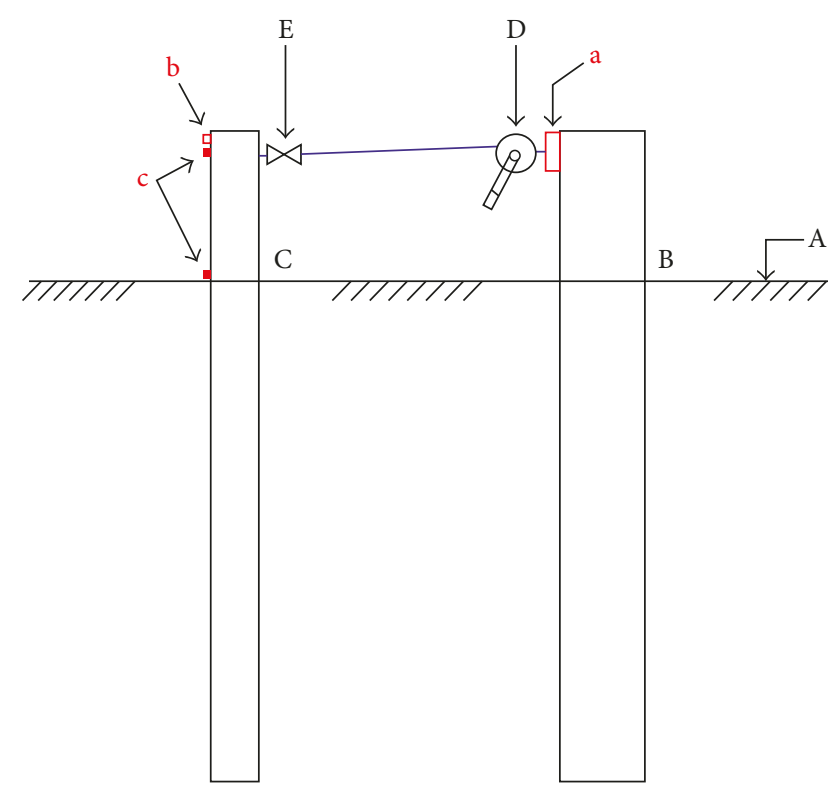

FIgURE 1: Elevation: test configuration for free-vibration response. The components are (A) soil surface, (B) reaction pile, (C) test pile, (D) chain hoist, (E) quick disconnect, (a) load cell, (b) accelerometer, and (c) displacement measurement. This sketch is not to scale.

(Fairbanks silt) are documented in a research report and a journal paper $[14,15]$. Preliminary investigation revealed a thin layer of gravel overlaying silt and a permafrost table located 20 to 22 feet $(6.096 \mathrm{~m}$ to $6.7056 \mathrm{~m})$ below ground surface.

A single-pile field test was conducted by using 25 -footlong $(7.62 \mathrm{~m}), 16$-inch diameter $(40.64 \mathrm{~cm})$ steel-jacketed reinforced concrete piles embedded approximate 20 feet $(6.096 \mathrm{~m})$ in silt. Eight Number 6 ASTM A706 reinforcing bars provided $1.93 \%$ of the cross-sectional area. Shear reinforcement consisted of Number 3 reinforcing bars bent into rings (or hoops) and spaced on 12 -inch $(30.48 \mathrm{~cm})$ centers outside of the longitudinal reinforcing bars; the outside diameter of each hoop was 1 inch $(2.54 \mathrm{~cm})$ less than the inside diameter of the steel pipe jacket. The pipe wall thickness is 0.375 inch $(0.9525 \mathrm{~cm})$. The purpose of this study was to evaluate pile behavior during winter month. The dynamic measurement was used to determine nature frequency and damping for the test pile.

The field test was performed in the month of January, and the ground temperature was $1.94^{\circ} \mathrm{F}\left(-16.7^{\circ} \mathrm{C}\right)$. The pile tops were a free end, a lateral load was applied to the pile, and the load was quickly released with a quick disconnect device. The accelerometer was used to measure how the pile vibrated once the load was released (Figure 1).

\section{Response Signal and Spectrum Analysis}

Figure 2 shows the measured acceleration signal. Figure 3 shows the FFT spectrum of this acceleration signal, and there are two specific peaks $f_{1}=101 \mathrm{~Hz}$ and $f_{2}=1072 \mathrm{~Hz}$. Figure 4 shows the spectrogram of the measured acceleration signal. From Figure 4(a), we can see the dominant component 


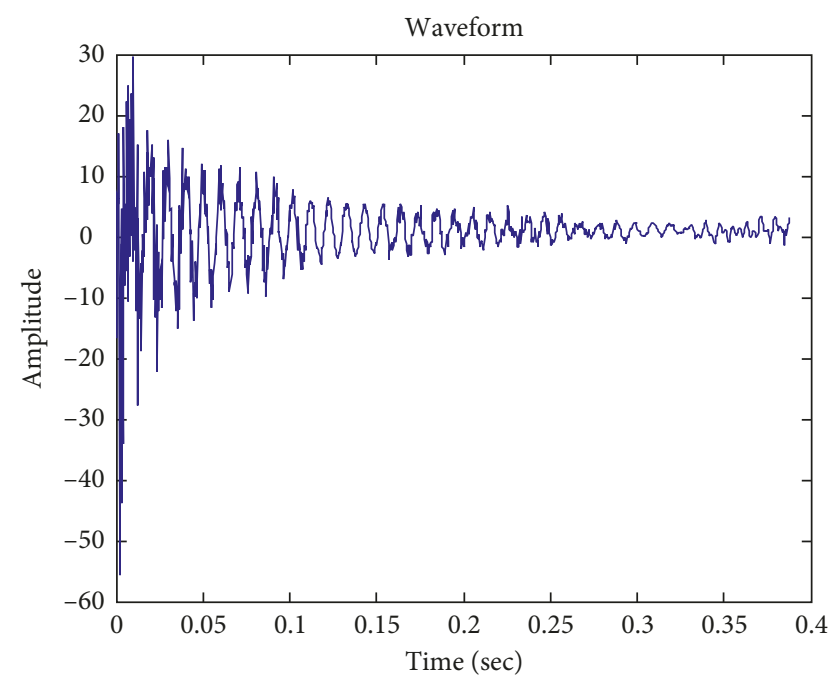

Figure 2: Time history of a measured acceleration signal.

corresponding to specific frequency $f_{1}=101 \mathrm{~Hz} ; f_{2}$ only exists a short period decay to zero after 0.1 second. This can be attributed as the initial nonlinear interaction of pile and soil; the other parts have harmonics and the variation of frequency which suggest the existence of nonlinear effect. Additional, from the 3D plot of Figure 4(b), we can see that the amplitude of the dominant component with specific frequency $f_{1}=101 \mathrm{~Hz}$ is substantially higher than the rest of components. The system's response can be approximated by this component.

\section{Parameter Identification by Using EEMD Method}

To accurately quantify the signal which has been roughly identified in the time-frequency spectrogram, this study used the ensemble empirical mode decomposition (EEMD) method which is developed based on the class empirical mode decomposition (EMD) method. Huang et al. [2] established empirical mode decomposition (EMD) which is a time-series demodulation method to extract a signal to a combination of intrinsic simple oscillatory signals (IMF) [8]. The original signal $x(t)$ is decomposed into $n$ intrinsic modes $c_{i}(t)$ and a residue $r_{n}(t)$. Assume that the original signal can be represented by

$$
x(t)=\sum_{i=1}^{n} c_{i}(t)+r_{n}(t)
$$

The detailed procedure and equations have been included in the published paper [8]. However, using this method, there exists a mode mixing problem. Huffman et al. [9] proposed a noise-assisted data analysis method referred to as ensemble EMD (EEMD). In brief, the steps of EEMD method are as follows: first, add white noise series $m_{i}(t)$ to the initial signal $x(t)$ :

$$
x_{i}(t)=x(t)+m_{i}(t) .
$$

Second, EMD is applied to decompose the signal to IMFs, $c_{j}(t)[9]$. Then, repeat the first step with different white

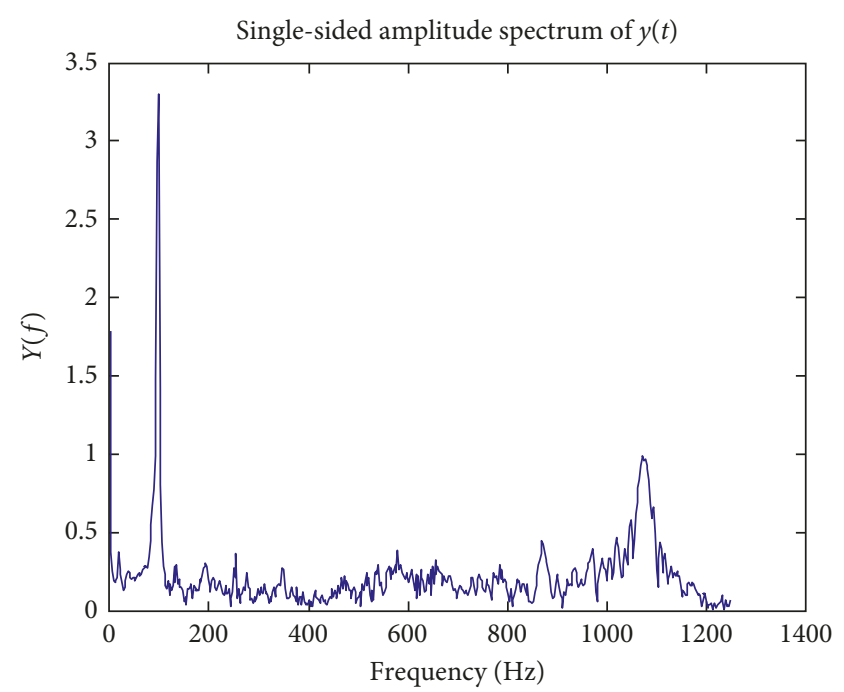

FIGURE 3: FFT of the vibration signal.

noises for each trail. Finally, estimate the means of corresponding IMF of the decompositions as the desired output:

$$
c_{j}(t)=\lim _{N \rightarrow \infty} \frac{1}{N} \sum_{i=1}^{N} c_{j i}(t),
$$

where $i$ is the ensemble number and $j$ is the component number [9]. Next, apply the Hilbert transform to all IMFs, to derive model parameters including frequency and damping:

$$
H\left[c_{j}(t)\right]=\frac{1}{\pi} \int_{-\infty}^{\infty} \frac{c_{j}(t)}{t-\tau} d \tau .
$$

After the Hilbert transform, $H\left[c_{j}(t)\right]$ and $c_{j}(t)$ form a complex signal $Z_{j}(t)$ :

$$
Z_{j}(t)=c_{j}(t)+i H\left[c_{j}(t)\right]=a(t) e^{i \varphi(t)} .
$$

Then, the envelop of every IMF can be given by

$$
\begin{aligned}
& a_{j}(t)=\sqrt{\left[c_{j}(t)\right]^{2}+H\left[c_{j}(t)\right]}, \\
& \varphi_{j}(t)=\arctan \left\{\frac{H\left[c_{j}(t)\right]}{c_{j}(t)}\right\},
\end{aligned}
$$

in which $a_{j}(t)$ the instantaneous amplitude of $x(t)$ reflects how the energy of $x(t)$ varies with time. The term $\varphi_{j}(t)$ is the instantaneous phase of $x(t)$. The instantaneous frequency $\omega(t)$ is defined as the time derivative of the instantaneous phase $\varphi(t)$ as follows:

$$
\omega(t)=\frac{d \varphi(t)}{d t} .
$$

Then the original signal $x(t)$ can be expressed as

$$
x(t)=\sum_{j=1}^{n} a_{j}(t) \exp \left[i \int \omega_{j}(t) d t\right] .
$$

In principle, the measured acceleration response of the pile, $\ddot{w}$, can be approximately decomposed by the EMD as follows: 


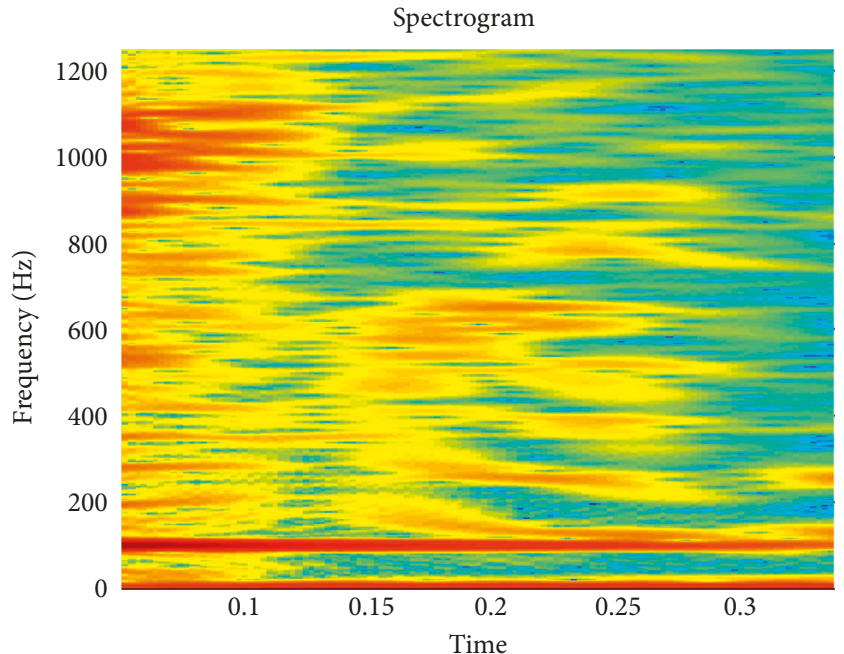

(a)

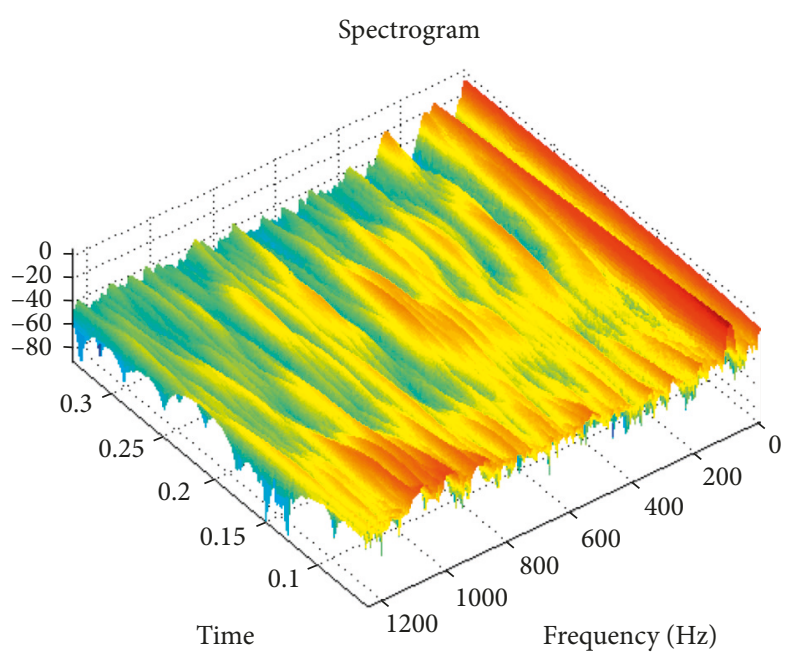

(b)

Figure 4: Spectrogram of a measured acceleration signal. (a) 2D plot and (b) 3D plot.

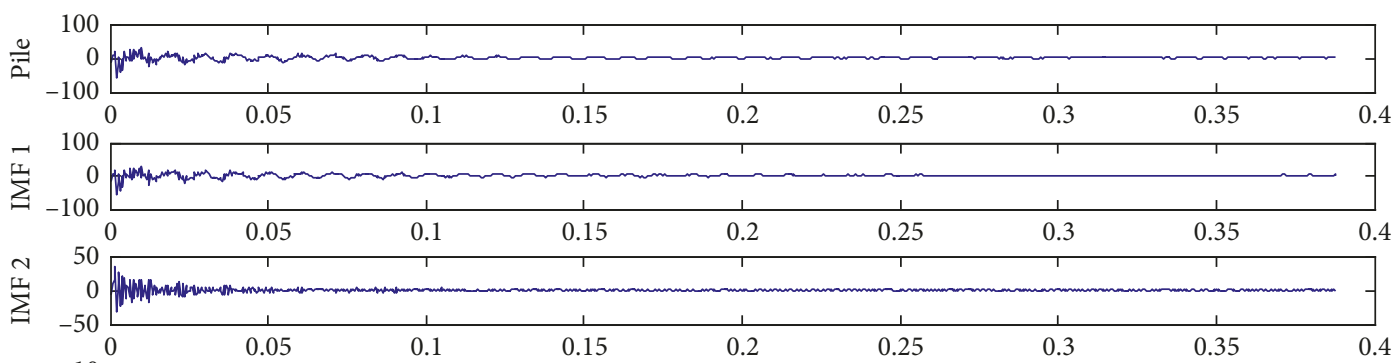

$\sum_{-10}^{m} \begin{array}{r}10 \\ \hline\end{array}$
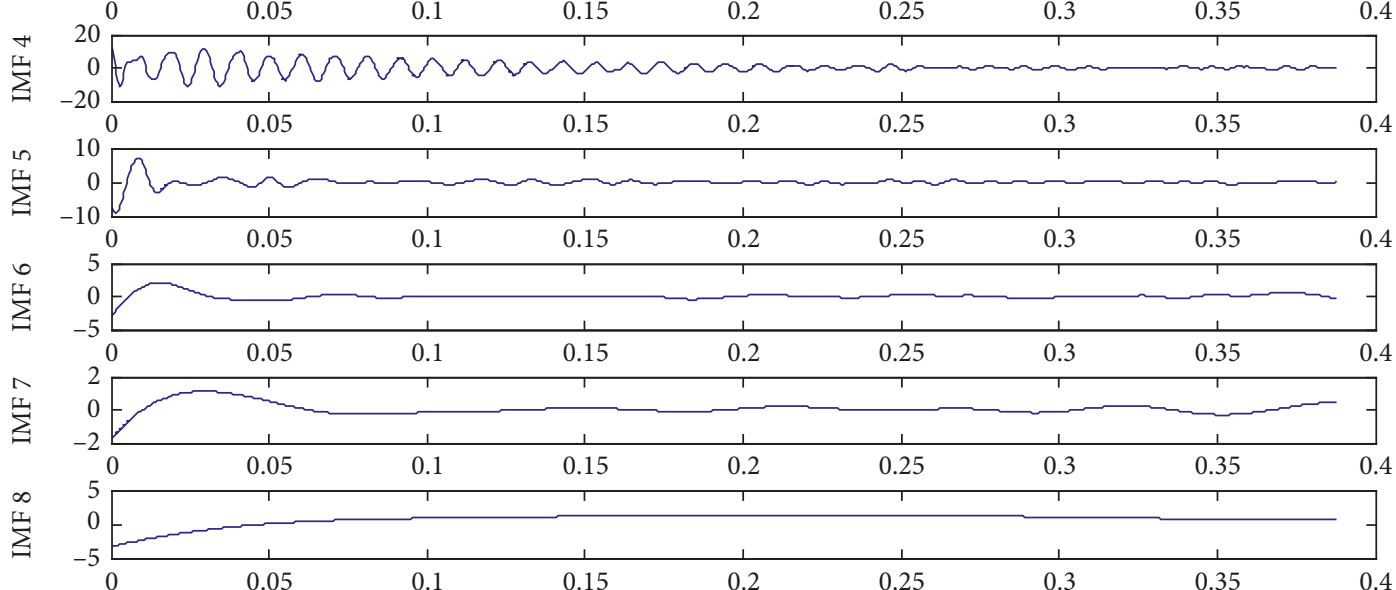

FIGURE 5: Decomposed components of a measured acceleration signal.

$$
\ddot{w}(t)=\sum_{j=1}^{k} \ddot{w}_{j}(t)+\sum_{i=1}^{n-k} c_{i}(t)+r_{n}(t),
$$

where $\ddot{w}_{j}(t)$ is the $j$ th modal acceleration response and $c_{i}(t)$ is the $i$ th IMF:

$$
\begin{aligned}
\omega_{j}(t) & =\omega_{d j} t-\theta_{j}, \\
\ln a_{i j} & =-\varsigma_{j} \omega_{j} t+\ln r_{i j} .
\end{aligned}
$$

Thus, the damped natural frequency $\omega_{d j}$ can be obtained from the slope of the phase angle plot in $\omega_{j}(t)$ versus $t$, and $\varsigma_{j}$ can be obtained from the slope of the plot in $\ln a_{i j}$ versus $t$.

The linear least-squares method can be used to fit the plots of $\ln a_{i j}$ versus time and $\omega_{j}(t)$ versus time. The following section illustrates the signal decomposition and parameter identification by using the measured acceleration 

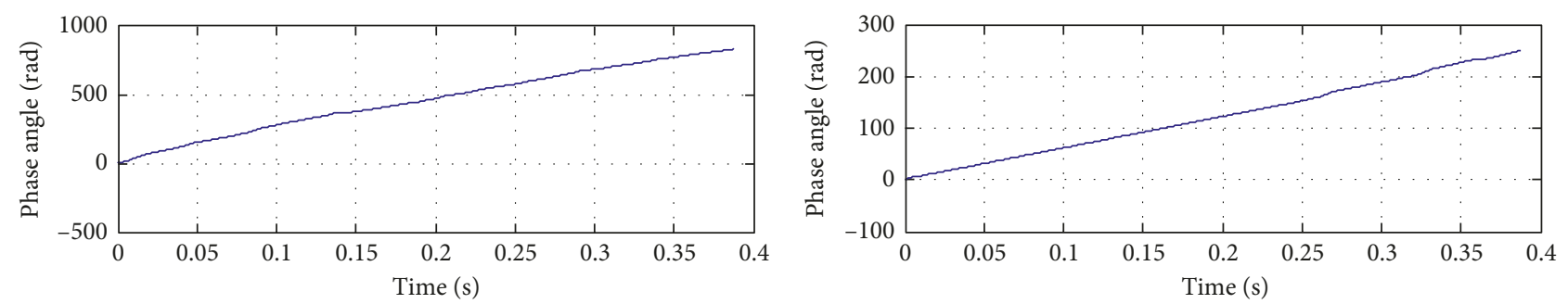

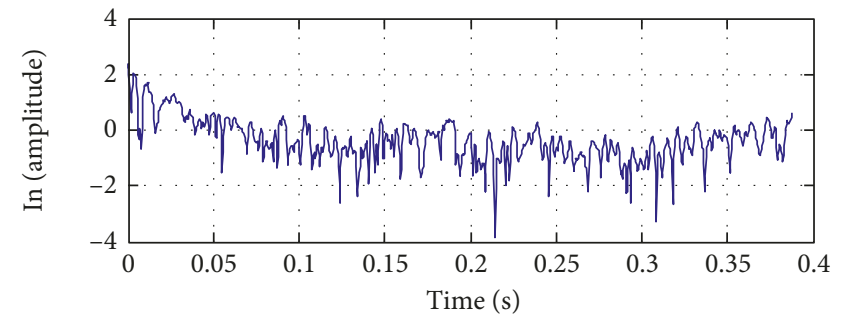

(a)

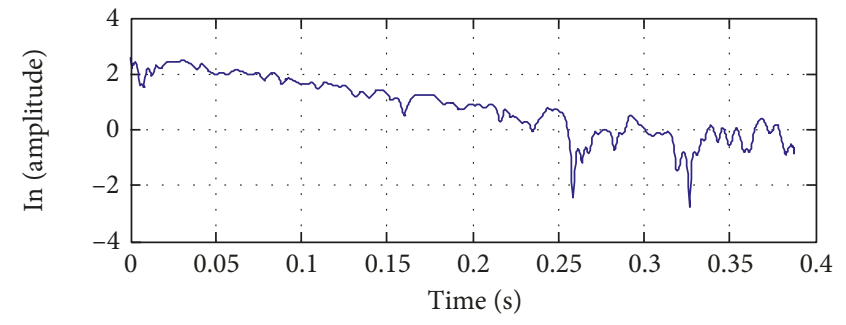

(b)

Figure 6: Characteristic plots of the decomposed signals corresponding to two specific frequencies: (a) $f_{2}=1072 \mathrm{~Hz}$ and (b) $f_{1}=101 \mathrm{~Hz}$.

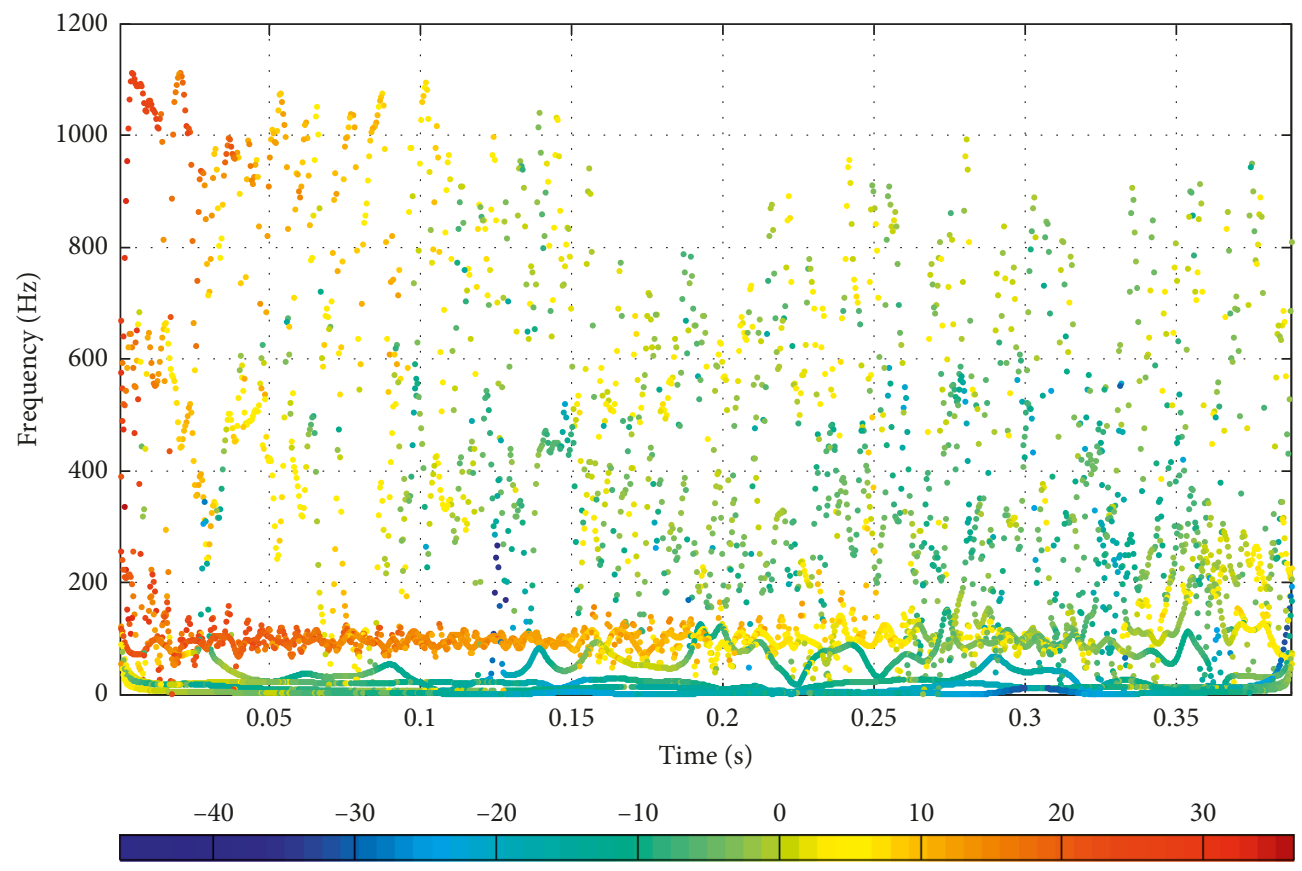

Power (dB)

FIGURE 7: Hilbert spectrum of intrinsic mode functions.

signal. Figure 5 shows the decomposed components of the measured acceleration signal (Figure 2).

The spectrum analysis of IMF2 and IMF4 demonstrated that their respective dominant peaks are $1072 \mathrm{~Hz}$ and $101 \mathrm{~Hz}$, respectively, which means IMF2 and IMF4 represent the two main natural modes $\left(f_{2}\right.$ and $\left.f_{1}\right)$ of the system, respectively.

In order to identify the dynamic properties of the decomposed signal, the obtained plots of phase angle and the logarithmic amplitude versus time for IMF2 and IMF4 are calculated and shown in Figure 6. The damped natural frequency can also be obtained from the slope of the phase angle plot, and the damping can be obtained from the slope of the amplitude versus $t$. The linear least-squares method can be used to fit the plots.

The damping coefficient corresponding to the mode $101 \mathrm{~Hz}$ is identified as $\varsigma_{1}=0.015$. And the damping coefficient corresponding to the mode $1072 \mathrm{~Hz}$ is identified as $\varsigma_{2}=0.035$. To further consider the complex frequency properties, the Hilbert spectrum is applied to retreat the acceleration signal recorded. Figure 7 shows the Hilbert spectrum of the decomposed signal intrinsic mode functions. From Figure 7, we can see that those respective components are well separated, and the timedependent properties of the frequencies are clearly shown. The dominant mode exhibits modulation centered at about $100 \mathrm{~Hz}$. 


\section{Conclusion}

This paper applied the EEMD method to evaluate the soilpile structure interaction under frozen condition. The method can decompose the vibration signal into different scales and help to identify the natural frequency and damping parameters accurately. This study identified the system parameter for the frozen soil-pile interaction. The test pile has two predominant natural frequencies: $101 \mathrm{~Hz}$ and $1072 \mathrm{~Hz}$, and the corresponding modal damping are 0.015 and 0.035 , respectively. The identified parameter can provide bridge engineering the required information for bridge design in a permafrost region. The application of EEMD has potential for other soil-pile vibration signals or other fields.

\section{Conflicts of Interest}

The authors declare that they have no conflicts of interest.

\section{References}

[1] K. Gröchenig, Foundations of Time-Frequency Analysis, Springer, New York, NY, USA, 2001, ISBN-13: 978-0135945322.

[2] N. E. Huang, Z. Shen, S. R. Long et al., "The empirical mode decomposition and the Hilbert spectrum for nonlinear and non-stationary time series analysis," Proceedings of the Royal Society of London A: Mathematical, Physical and Engineering Sciences, vol. 454, no. 1971, pp. 903-995, 1998.

[3] Z. Wu and N. E. Huang, "Ensemble empirical mode decomposition: a noise-assisted data analysis method," Advances in Adaptive Data Analysis, vol. 1, no. 1, pp. 1-41, 2009.

[4] Z. J. Yang, Q. Li, J. Horazdovsky, J. L. Hulsey, and E. E. Marx, "Performance and design of laterally loaded piles in frozen ground," Journal of Geotechnical and Geoenvironmental Engineering, vol. 143, no. 5, p. 06016031, 2016.

[5] S. Sritharan, D. J. White, and M. T. Suleiman, "Bridge column foundation-soil interaction under earthquake loads in frozen conditions," in Proceedings of the 13th World Conference on Earthquake Engineering, pp. 1-6, Vancouver, Canada, August 2004.

[6] M. T. Suleiman, S. Sritharan, and D. J. White, "Cyclic lateral load response of bridge column-foundation-soil systems in freezing conditions," Journal of Structural Engineering, vol. 132, no. 11, pp. 1745-1754, 2006.

[7] Z. J. Yang, U. Dutta, D. Zhu, E. Marx, and N. Biswas, "Seasonal frost effects on the soil-foundation-structure interaction system," Journal of Cold Regions Engineering, vol. 21, no. 4, pp. 108-120, 2007.

[8] E. Mohtashami and A. Shooshtari, "A multimode adaptive pushover procedure for seismic assessment of integral bridges," Advances in Civil Engineering, vol. 2013, Article ID 941905, 13 pages, 2013.

[9] J. T. Huffman, F. Xiao, G. Chen, and J. L. Hulsey, "Detection of soil-abutment interaction by monitoring bridge response using vehicle excitation," Journal of Civil Structural Health Monitoring, vol. 5, no. 4, pp. 389-395, 2015.

[10] W. Caesarendra, P. B. Kosasih, A. K. Tieu, C. A. Moodie, and B. K. Choi, "Condition monitoring of naturally damaged slow speed slewing bearing based on ensemble empirical mode decomposition," Journal of Mechanical Science and Technology, vol. 27, no. 8, pp. 2253-2262, 2013.

[11] M. C. Chen, L. S. Chen, and Y. Wei, "Apply ensemble empirical mode decomposition to discover time variants of metro station passenger flow," in Proceedings of the 4th International Conference on Industrial Engineering and Applications (ICIEA), pp. 239-243, Nagoya, Japan, April 2017.

[12] B. Zhu and J. Chevallier, A Multiscale Analysis for Carbon Price with Ensemble Empirical Mode Decomposition, Pricing And Forecasting Carbon Markets, Springer International Publishing, Basel, Switzerland, 2017.

[13] D. C. Furlaneto, L. S. Oliveira, D. Menotti, and G. D. C. Cavalcanti, "Bias effect on predicting market trends with EMD," Expert Systems with Applications, vol. 82, pp. 19-26, 2017.

[14] Z. Yuanlin and D. L. Carbee, Creep and Strength Behavior of Frozen Silt in Uniaxial Compression, Cold Regions Research and Engineering Laboratory, Hanover, NH, USA, 1987.

[15] F. Xiao, G. S. Chen, J. L. Hulsey, J. D. Dolan, and Y. Dong, "Ambient loading and modal parameters for the Chulitna River Bridge," Advances in Structural Engineering, vol. 19, no. 4, pp. 660-70, 2016. 


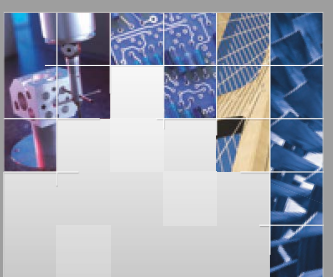

\section{Enfincering}
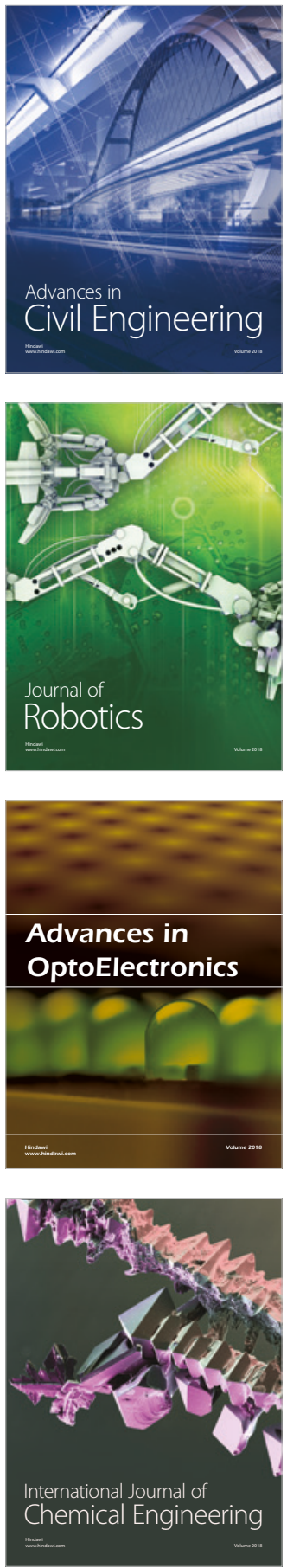

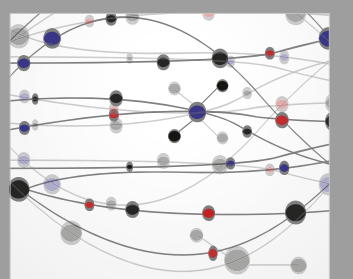

\section{Rotating \\ Machinery}

The Scientific World Journal

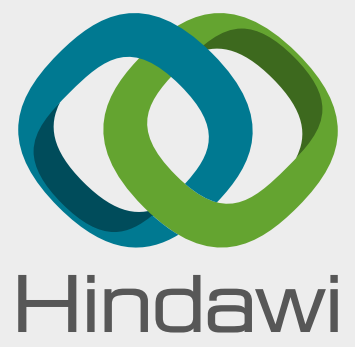

Submit your manuscripts at

www.hindawi.com
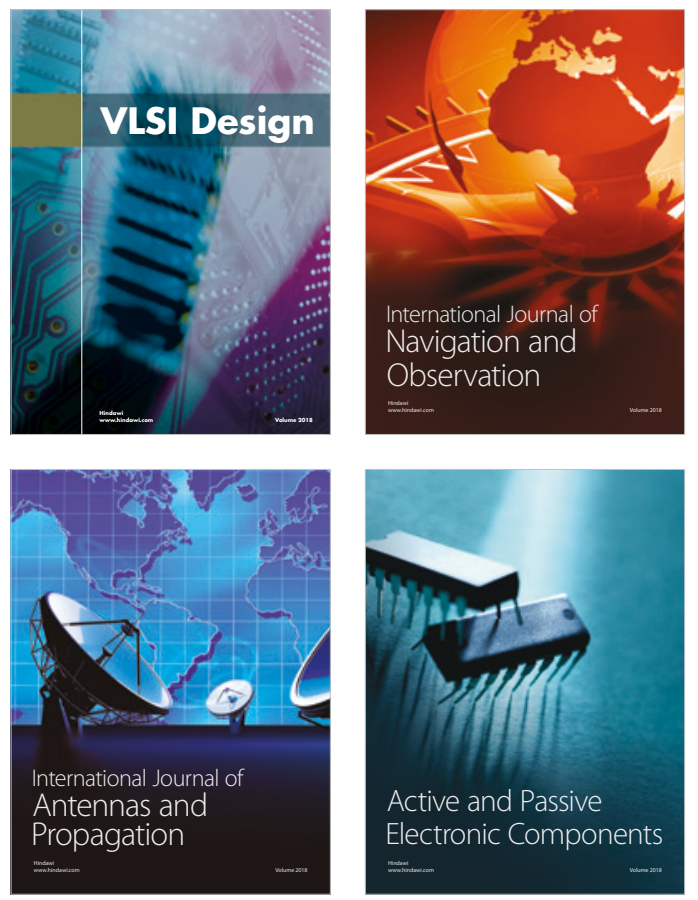
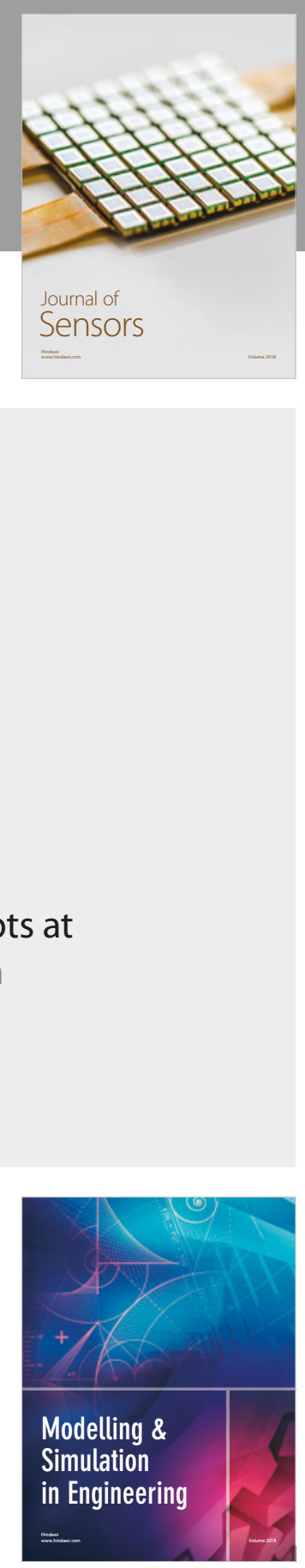

\section{Advances \\ Multimedia}
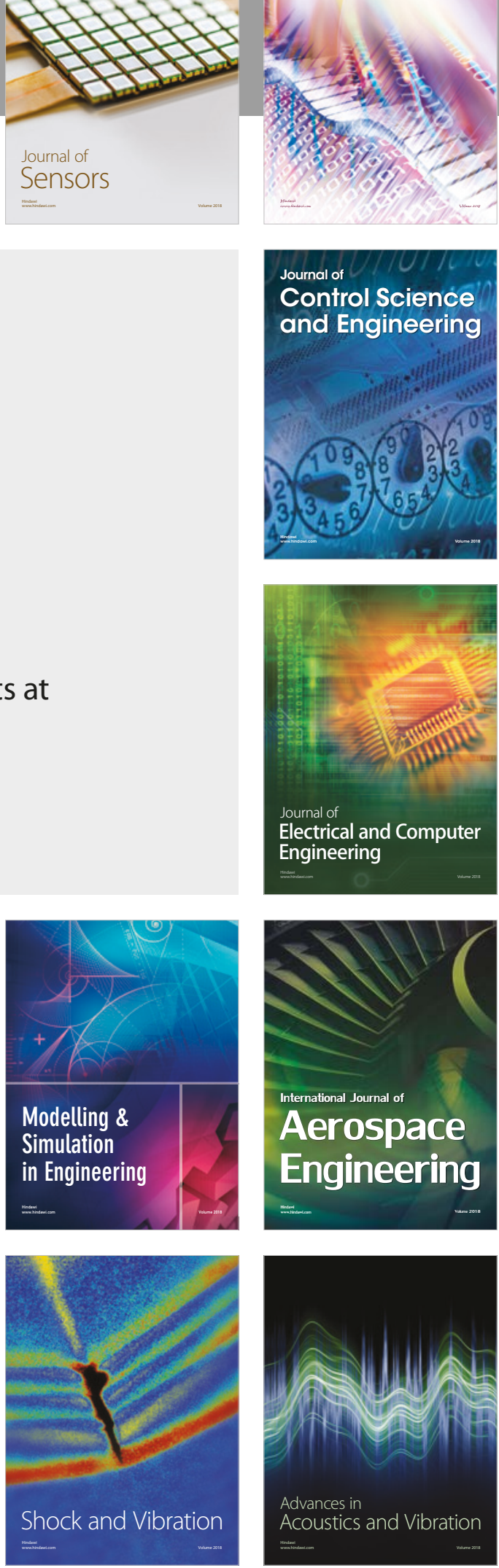